\title{
A loaded self-managed exercise programme for patellofemoral pain: a mixed methods feasibility study
}

Benjamin E. Smith ${ }^{1,2^{*}}$ D, Paul Hendrick ${ }^{3}$, Marcus Bateman ${ }^{1}$, Fiona Moffatt ${ }^{3}$, Michael Skovdal Rathleff ${ }^{4,5}$, James Selfe ${ }^{6}$, Toby O. Smith ${ }^{7}$ and Pip Logan²

\begin{abstract}
Background: A novel loaded self-managed exercise programme that includes pain education and self-management strategies may result in better outcomes for people with patellofemoral pain (PFP). However, establishing program feasibility is an essential first step before testing efficacy. The purpose of this study was to evaluate the feasibility and acceptability of conducting a definitive RCT which will evaluate the clinical and cost-effectiveness of a loaded selfmanaged exercise programme for people with PFP compared with usual physiotherapy.
\end{abstract}

Methods: In a mixed methods, pragmatic, randomised controlled feasibility study, 60 participants with PFP (57\% female; mean age 29 years) were recruited from a physiotherapy clinic within a large UK teaching hospital. They were randomly allocated to receive either a loaded self-managed exercise programme $(n=30)$ or usual physiotherapy $(n=30)$. Feasibility indicators of process, resources, and management were collected through follow-up of standardised questionnaires six months after recruitment and semi-structured interviews with 20 participants and physiotherapists.

Results: Recruitment rate was 5 participants per month; consent rate was 99\%; adherence to intervention appointments was $87 \%$; completeness of questionnaire data was 100\%; and adherence to intervention delivery was $95 \%$.

Three exercise diaries were returned at six months (5\%). At six months, 25 questionnaire booklets were returned (9 in the loaded self-managed group, 16 in the usual physiotherapy group), with a total retention rate of $42 \%$.

At six months, 56\% (5/9) of respondents in the loaded self-managed group and 56\% (9/16) in the usual physiotherapy group were classified as 'recovered'.

Both groups demonstrated improvements in average pain (VAS), kinesiophobia, pain catastrophizing, general self-efficacy and EQ-5D-5 L from baseline to six months.

Conclusion: The results of this feasibility study confirm that it is feasible and acceptable to deliver a loaded self-managed exercise programme to adults with PFP in an NHS physiotherapy outpatient setting. However, between group differences in lost to follow up and poor exercise diary completion mean we are uncertain on some feasibility aspects. These methodological issues need addressing prior to conducting a definitive RCT.

Trial registration: ISRCTN 35272486. Registered 19th December 2016.

Keywords: Mixed-methods study, Feasibility, Patellofemoral pain, Anterior knee pain, Exercise therapy

\footnotetext{
* Correspondence: benjamin.smith3@nhs.net

'Physiotherapy Department (Level 3) London Road Community Hospital, University Hospitals of Derby and Burton NHS Foundation Trust, Derby DE1 2QY, UK

${ }^{2}$ Division of Rehabilitation and Ageing, School of Medicine, University of Nottingham, Nottingham, UK

Full list of author information is available at the end of the article
}

(c) The Author(s). 2019 Open Access This article is distributed under the terms of the Creative Commons Attribution 4.0 International License (http://creativecommons.org/licenses/by/4.0/), which permits unrestricted use, distribution, and reproduction in any medium, provided you give appropriate credit to the original author(s) and the source, provide a link to the Creative Commons license, and indicate if changes were made. The Creative Commons Public Domain Dedication waiver (http://creativecommons.org/publicdomain/zero/1.0/) applies to the data made available in this article, unless otherwise stated. 


\section{Background}

Patellofemoral pain (PFP) is one of the most common forms of knee pain in adults under the age of 40 [1-3]. It has an estimated prevalence of $23 \%$ in the general population [4]. The long-term prognosis for PFP is poor [5]. Only one-third of patients are pain-free one year after diagnosis [5], and 91\% still report pain and dysfunction four years post-diagnosis [6].

There remains scientific debate around the underlying aetiology of the condition [7]. It is thought most likely to be multifactorial in origin [8]. There is currently little high-quality level 1 evidence to base conservative management on [9]. Even in relation to exercise therapy, which has the strongest evidence-base [9], there remains insufficient evidence to determine the best form and dose of exercise [10].

Many patients with PFP develop associated pain- related fear, such as fear-avoidance, catastrophizing thoughts and low self-efficacy [11-14]. These psychological factors not only affect function and quality of life of patients with pain but can modulate the individual pain experience. This may influence the development or maintenance of chronic pain states [15-21]. A systematic review of self-management interventions for chronic musculoskeletal (MSK) pain (16 studies; $n=4047$ ), found self-efficacy and depression were the strongest prognostic factors for pain and disability (irrespective of the intervention) [22]. Pain catastrophizing and increasing physical activity were the strongest mediating factors [22]. This provides a foundation that interventions should be aimed at improving pain-related fear and increasing physical activity in relation to self-management strategies.

Exercise therapies designed to load and temporarily aggravate patients' symptoms have demonstrated improvements for a range of MSK disorders including tendon pain [23], shoulder pain [24-26], low back pain $[27,28]$ and plantar heel pain [29]. The potential rationale and mechanisms behind loaded painful exercises include positive changes to central and peripheral pain mechanisms, the immune system and affective aspects of pain [30]. Specifically, these exercises are prescribed to address pain-related fear within a framework of 'hurt not equalling harm', with the pain experience rationalised as a consequence of 'de-conditioned' tissue. Hypothetically, over time, the exercises reduce pain related-fear and the overall sensitivity of the central nervous system, with a modified pain experience [30, 31].

Exercise interventions for PFP have shown a 'dose-response', where the greater the volume and intensity of exercise the patient performs the greater their improvement in long-term pain and function [32, 33]. A recent systematic review of painful exercises versus pain-free exercises for chronic MSK pain found regimes using painful exercises offered a small, but significant benefit over pain-free exercises in the short-term. It also reported that regimes using painful exercises typically have higher loads and dose of exercise [31]. Nonetheless, the optimal dose of exercise for the greatest improvements in PFP remains uncertain [10].

Based on these uncertainties, high-quality research on exercise prescription in relation to pain mechanisms and response to load/resistance warrants further investigation. However, to ensure the success of a large multi-centred randomised controlled trial (RCT), several feasibility questions needed to be answered.

The primary aim of this study was to establish the feasibility and acceptability of conducting a definitive RCT which will evaluate the clinical and cost-effectiveness of an intervention based on pain science (where exercises are designed to load and temporarily aggravate patients' symptoms), self-management strategies and improvements in physical activity levels for people with PFP compared to usual physiotherapy. The intervention has been referred to as a loaded self-managed exercise programme.

\section{Methods}

This study was reported in accordance with the Consolidate Standard of Reporting Trials (CONSORT) statement [34] and Template for Intervention Description and Replication guidelines (TiDieR) [35].

The protocol was approved by the West Midlands Black Country Research Ethics Committee (ref: 16/WM/ 0414) and sponsored by University Hospitals of Derby and Burton NHS Foundation Trust. A full description of the methods has been previously published [36]. A brief description is detailed below.

\section{Study design}

A pragmatic, randomised controlled, single-centre, feasibility study, with an embedded qualitative component.

\section{Participants}

Participants were recruited between February 2017 and January 2018 from a physiotherapy waiting list at a large NHS teaching hospital. Patients were referred from general practitioners and from orthopaedics and rheumatology hospital departments. An introductory letter accompanied by an information sheet and consent form was sent out to potential trial participants by a member of the clinical team. This was followed up by a telephone call from a member of the clinical team offering further information and enquiring about participation. Inclusion and exclusion criteria followed currently accepted criteria [37] and were checked both verbally (by telephone initially, then face to face by the same physiotherapist with 10 years' MSK experience) 
(Table 1). The same physiotherapist took consent, before baseline data was taken and then randomisation.

\section{Sample size}

Sixty participants were planned to be recruited; 30 participants per group. The qualitative component interviewed a purposive sample of 20 participants; 10 treating physiotherapists (five from each group), and ten patients (five from each group).

A formal sample size calculation was not performed since the study was designed as a feasibility study. Sample sizes between 24 [38] and 50 [39] have been recommended as providing suitable data for performing a sample size calculation. Therefore accounting for an attrition rate of $20 \%$, these sample sizes were chosen for the feasibility RCT to provide sufficiently robust, meaningful amounts of information [40].

\section{Randomisation}

Patients were randomised to either the intervention group (loaded self-managed exercises) or the control group (usual physiotherapy) (1:1) by a web-based randomisation service with secure password protected login using random variable block-size.

Due to the nature of therapeutic interventions, blinding of the participants and physiotherapists was not possible [41], and participants were aware of the purpose of the study. All participants were blinded to the criteria for feasibility.

\section{Interventions}

\section{Training of the physiotherapists}

The training package was delivered to the treating physiotherapists by the research team. The training package was designed to be easily deliverable and in a short space of time. It consisted of two, two-hour training

Table 1 Participant eligibility criteria

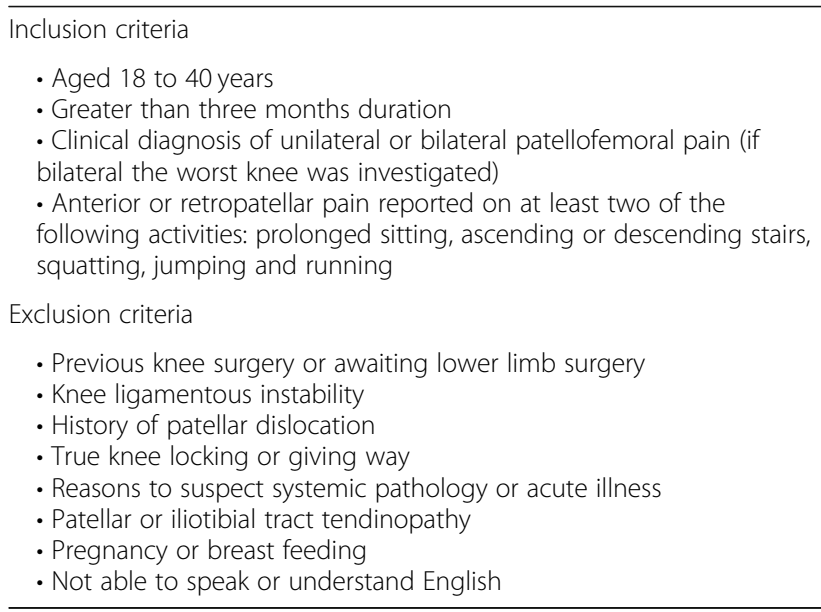

sessions, scheduled to fit into the department's usual in-service training slots.

The first session delivered to all physiotherapists consisted of: background to PFP, rationale for further study, overview of research design, clinical equipoise, usual physiotherapy, discussion and, questions and answers.

The second training session, delivered only to physiotherapists delivering the loaded self-managed intervention, consisted of: revision of training session one, pain education, the loaded exercise, self-management strategies, discussion and, questions and answers.

All physiotherapists were supported to continue giving the interventions through weekly informal chats.

\section{Loaded self-managed exercise programme}

The 'experimental' intervention was a loaded selfmanaged exercise programme. It is a novel intervention based on pain science (where a single exercise is designed to load and temporarily aggravate patients' symptoms), self-management strategies and improvements in physical activity levels, delivered by trained and supported NHS physiotherapists [36]. The intervention was set within a framework of reducing fear-avoidance, with an emphasis on participant self-management of the condition and exercise programme, and improvements in physical activity levels [36]. A full description of the intervention has been previously published [36]. A brief description is detailed below.

Education of the patients regarding pain mechanisms, such as addressing any beliefs or fear within the participant that pain was a sign of tissue damage, was planned to take up a large portion of the clinical time. Patient discourse regarding tissue-based pathology models of pain, e.g. patellar mal-tracking, or limb mal-alignment was actively discouraged by the physiotherapist.

The physiotherapists prescribed the exercise and typically involved body weight resistance in the form of a modification of the 'Step Down' function test [42], a single leg squatting exercise sideways on a step. The exercise required balance, knee extension strength, eccentric control and isometric hip strength. The participants were advised to exercise to the point of fatigue, such that it reproduced their pain and discomfort, whilst ensuring pain was manageable [43-45].

Exercise progression was guided by the symptomatic response. Participants were advised that on cessation of the exercise, the pain should remain no worse than pre-exercise [43]. Participants with more severe pain were able to start on a lighter regime, guided by the baseline functional assessment by the treating physiotherapist. Regression of the exercise programme was reduced repetitions or lightening the exercise, for example moving from single-leg squats to double-leg 
squats or isometric static squats. Progression was in the form of increased repetitions or increasing the load by moving to plyometric exercises, such as jumping and hopping, for younger participants with higher sporting requirements.

Participants were taught one exercise, to aid adherence [46], whilst being time-efficient [47]. Participants were advised to perform the exercise twice daily. They were encouraged to self-direct progressing/regressing the repetitions, as guided by their pain response. This was done to promote internalising the locus of control and towards self-management and overall improvements in physical activity levels [48].

Self-management strategies employed included: goal-setting, discussions about managing 'flare ups' and potential or perceived barriers to successful outcomes of the intervention $[47,49]$.

Keeping the treatment pragmatic, the timing of follow-ups, number of treatment sessions, frequency and discharge, and physiotherapy-led passive treatments was at the discretion of the qualified physiotherapist. However, as the aim of the programme was focused on self-management strategies, self-directed exercises were promoted and concomitant treatments discouraged. All participants had the opportunity to telephone for support if required, as per usual department practice.

To avoid cross-contamination between the two groups, the intervention group was delivered by physiotherapists who were excluded from treating participants from the control group (and vice versa).

\section{The comparator}

The comparator was usual physiotherapy as directed by the clinical judgement of the treating physiotherapist [50]. Usual physiotherapy often involves strengthening exercises, taping, stretches, foot orthoses, movement retraining and is typically aimed at reducing load on the patella and avoidance of painful exercise and activity $[9,50]$.

\section{Outcomes}

The following outcomes were measured.

\section{Feasibility outcomes}

\section{Recruitment \& eligibility}

Recruitment rates were recorded and defined as the number of participants recruited each month, compared with expected and feasible recruitment rates. We had expected to be able to recruit 4.6 patients per month, based on estimates on the referral rate which was observed in the department between January 2013 and October 2013.

The consent rate was calculated by dividing the number of individuals who met inclusion criteria, by the number who consented to participate in the study.

\section{Randomisation \& blinding}

Randomisation was assessed on the rate of participants randomised after consent, and on any challenges reported by the recruiting researcher. Baseline demographic data of age, sex and duration of symptoms were collected.

\section{Adherence \& acceptability}

Compliance levels with the intervention were monitored through a participant activity diary. Participants were asked to complete an exercise diary daily for six months to indicate how many exercise repetitions they had completed each day. Adherence to treatment was assessed by the adherence rate to treatment (\%) from exercise diaries returned at six months, calculated by the percentage of days they indicated they completed their exercise(s). Adherence to appointments (\%) was based on the number of 'did not attend' (DNAs), where a participant fails to attend their physiotherapy appointment.

\section{Patient-reported outcome measures}

The retention rate / lost at follow-up was assessed on the percentage of returned outcome forms at three and six months. The percentage of missing data was also recorded.

\section{Resources \& study management}

Participant processing time was measured as the number of days from initial contact (information letter being sent) to consent and randomisation.

Fidelity was defined as adherent and competent delivery of the intervention, and was evaluated by analysis of the physiotherapists' clinical notes against a three-point checklist outlining important details and components of intervention to be completed by the physiotherapist. The three-point checklist included: specific pain education; delivery of a loaded exercise programme; and discussion on self-management strategies, which, as previously mentioned, where main topics discussed during the intervention training. This analysis was conducted for both the loaded self-managed, and usual physiotherapy groups, with a full analysis of treatments delivered in the usual physiotherapy group.

\section{Patient-reported outcome measures}

Clinical outcome measures were collected at baseline, three and six months post-randomisation. The follow-up outcome measures were posted to the patients' home, with a pre-paid enveloped to return.

The primary outcome measure was the global rating of change (GROC) at follow-up, the proportion of participants who had recovered (defined as 'completely recovered' or 'strongly recovered'), measured on a seven-point 
Likert scale ranging from 'completely recovered' to 'worse than ever' $[33,51,52]$.

Secondary outcome measures included: the visual analogue scale (VAS) for pain, average over the last week [53], the Tampa Scale for Kinesiophobia (TSK) [11, 54], the 'Pain Catastrophizing Scale' (PCS) [55], the General Self Efficacy Scale (GSES) [56], and the generic health outcome Euro-QOL using UK dataset (EQ-5D-5 L), which included a general health VAS [57]. Participation in leisure time sport or exercises within a week was also recorded. The occurrence of an adverse event as a result of participation within this study was not expected, and therefore no adverse event data were collected.

Participants who had not returned the questionnaires were telephoned after seven days to encourage them to complete and return these.

\section{Embedded qualitative interviews}

A sample of 20 participants from the cohort were interviewed. Purposive sampling was employed to gain maximum variation in participants. Ten patients were selected based on the population in terms of intervention groups (both the loaded self-managed group and usual physiotherapy group), age and gender. Attempts were made to include patients who failed to return clinical outcome measures and patients who were classed as non-responders to the treatment (based on the clinical outcome measures) in both groups.

Ten physiotherapists (five from each group) were also interviewed. A purposive sample was selected based on certain characteristics to represent a spectrum population in terms of intervention groups, age, sex and length of time qualified.

Interviews were semi-structured and broadly considered the acceptability and feasibility of study design and training package delivered to physiotherapists.

\section{Data analysis}

\section{Quantitative data}

Reflecting a feasibility study design [58], descriptive statistics along with point estimates, confidence intervals (95\%), and effect sizes using independent t-tests, were presented for all appropriate clinical outcome measures. Participant characteristics were presented using means, standard deviations and ranges for quantitative variables and counts and proportions for categorical variables. Feasibility outcomes were described using descriptive statistics. Sensitivity analysis of the primary outcome measure, GROC, was carried out, looking at the proportion of participants who had recovered defined as 'completely recovered,' 'strongly recovered' or 'slightly recovered'.

Statistical analysis was undertaken using SPSS version 24.0 (Armonk, NY: IBM Corp). No data imputation was performed to account for missing data; intention-to-treat with complete-case analysis was conducted. As recommended by the CONSORT statement, statistical comparison of baseline data was not performed [59].

Feasibility thresholds, as agreed a priori [36], were set at $75 \%$ to assess reliability and completeness of outcome measures and used to indicate either success or if strategies are required to improve the viability of any future definite trial, these are presented in Table 2, with feasibility results summarised in Table 3 . Where it was not possible to use quantitative data to demonstrate success, outcomes were reported narratively.

\section{Qualitative data}

The qualitative component followed a thematic analysis approach, as described by Braun and Clarke (2006) [60]. Full details on the qualitative analysis used in this study have previously been published [14].

\section{Results}

\section{Feasibility outcomes}

Recruitment \& eligibility

Recruitment rate was 5.0 participants per month over a 12-month period, exceeding the recruitment rates for feasibility. See Fig. 1 for recruitment rate comparisons, and Fig. 2 for the flow of participants through the study. Over 12-months, 185 referrals were reviewed as potentially eligible, 185 recruitment packs were posted and five (3\%) 'opt out' slips were returned.

\section{Randomisation \& blinding}

The recruiting researcher reported no randomisation issues. All consenting participants were randomised. There were no baseline imbalances for demographics, baseline symptoms and clinical outcome measures (Table 4).

\section{Adherence \& acceptability}

Only one exercise diary (3\%) was returned from participants in the loaded self-managed group. This indicated

Table 2 Thresholds for feasibility outcomes

\begin{tabular}{lll}
\hline Outcome & Indicator & Successful \\
\hline Recruitment \& eligibility & $\begin{array}{l}\text { Recruitment rate (participants } \\
\text { per month) } \\
\text { Consent rate (\%) }\end{array}$ & $>3.75$ \\
$\begin{array}{l}\text { Adherence \& } \\
\begin{array}{l}\text { acceptability } \\
\text { Outcome measures }\end{array}\end{array}$ & $\begin{array}{l}\text { Adherence to appointments (\%) } \\
\text { Retention rate (\%) }\end{array}$ & $>75$ \\
Resources \& study & $\begin{array}{l}\text { Adherence to intervention } \\
\text { management }\end{array}$ & $>75$ \\
\hline
\end{tabular}


Table 3 Thresholds for feasibility outcomes - results

\begin{tabular}{|c|c|c|c|c|c|}
\hline Outcome & Indicator & Successful & Result & Feasible & Suggested modifications \\
\hline \multirow[t]{2}{*}{ Recruitment \& eligibility } & $\begin{array}{l}\text { Recruitment rate (participants per } \\
\text { month) }\end{array}$ & $>3.75$ & 5.0 & Yes & \\
\hline & Consent rate (\%) & $>75$ & 98.6 & Yes & \\
\hline Adherence \& acceptability & Adherence to appointments (\%) & $>75$ & 86.8 & Yes & \\
\hline \multirow[t]{2}{*}{ Outcome measures } & Retention rate (\%) & $>75$ & 41.7 & No & $\begin{array}{l}\text { Reduce the number of outcome measures, } \\
\text { use of IT (e.g. text message), improve } \\
\text { communication between treating } \\
\text { physiotherapist and participant, relax criteria } \\
\text { for success, entry into a prize draw, telephone } \\
\text { consultations. }\end{array}$ \\
\hline & Completeness of data (\%) & $>75$ & 99.7 & Yes & \\
\hline $\begin{array}{l}\text { Resources \& study } \\
\text { management }\end{array}$ & Adherence to intervention delivery (\%) & $>75$ & 94.9 & Yes & \\
\hline
\end{tabular}

exercise adherence of $40 \%$ of the time. Two exercise diaries from participants in the usual physiotherapy group (7\%) were returned, with a mean adherence rate of $43 \%$.

Adherence to appointments was $87 \%$; with $79 \%$ in the loaded self-managed exercise group and $92 \%$ in the usual physiotherapy group. The treatments provided by the treating physiotherapists can be seen in Table 5 .

\section{Outcome measures}

At three month follow-up, participants who had not returned questionnaires were telephoned and reminded. At this stage, one participant (3\%) from the loaded self-managed exercise group withdrew, and two participants $(7 \%)$ from the usual physiotherapy group withdrew.

Differences in lost to follow-up were demonstrated with the return rate of participants' questionnaire booklets. At three months, 27 questionnaire booklets were returned (nine in the loaded self-managed group, 18 in usual physiotherapy), with a total retention rate of $45 \%$. At six months, 25 questionnaire booklets were returned (nine in the loaded self- managed group, 16 in usual physiotherapy), with a total retention rate of $42 \%$.

Of the returned forms, all were completed fully, apart from one participant omitting the Tampa Scale for

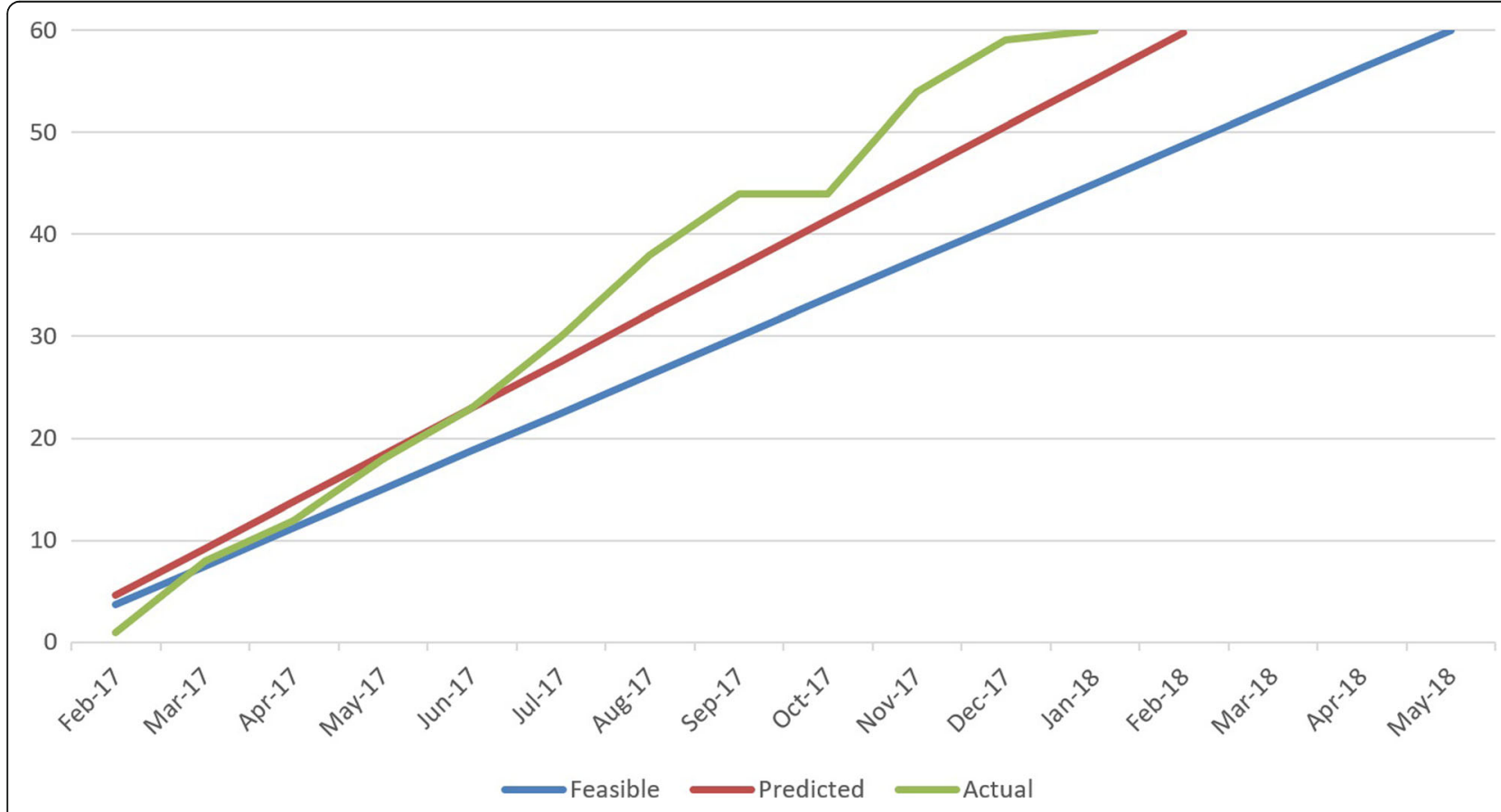

Fig. 1 Actual recruitment rate, compared to feasible and predicted 


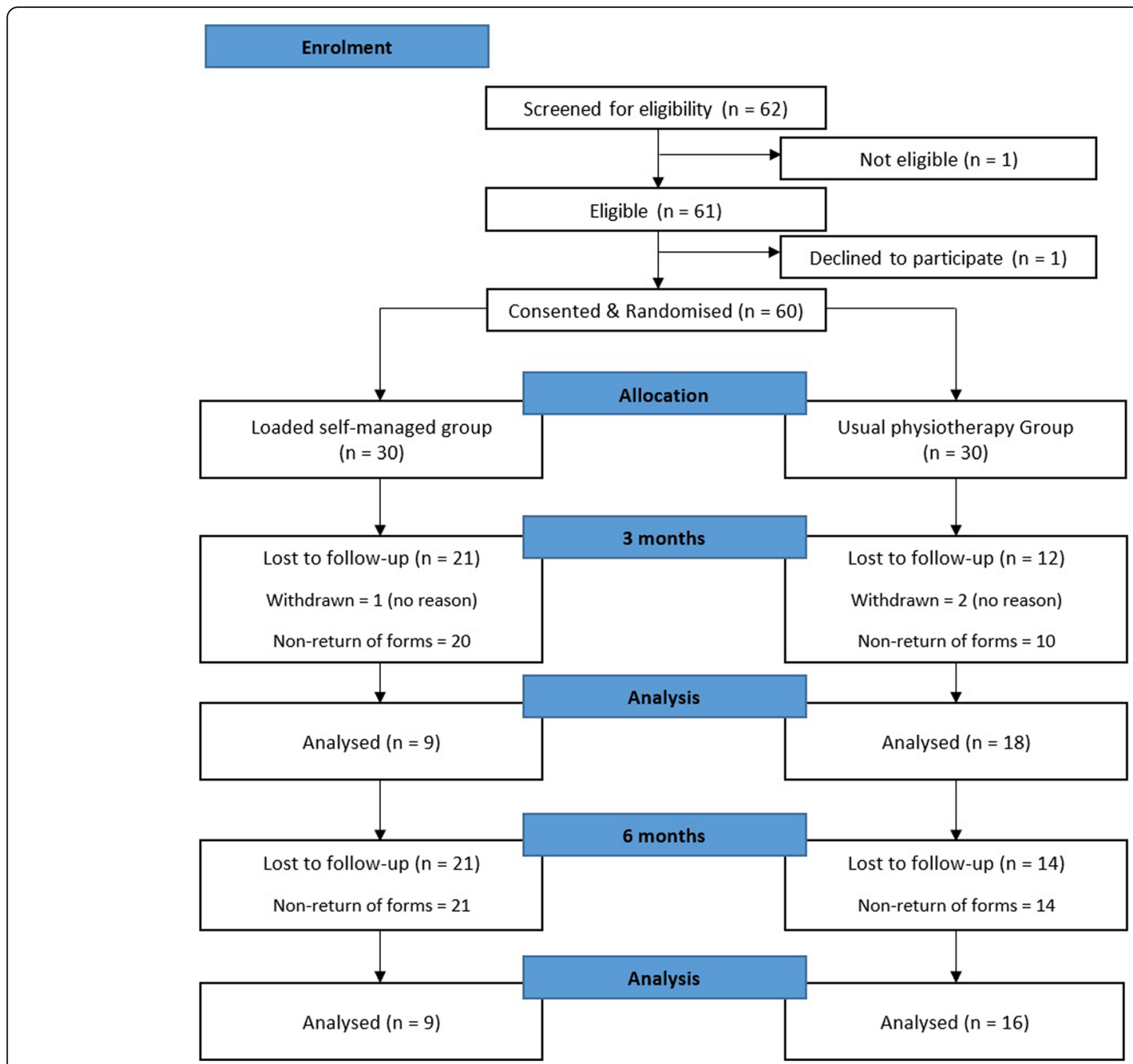

Fig. 2 Flow of participants through the study

Kinesiophobia at three months, with completeness of data indicators of $99.7 \%$.

\section{Resources \& study management}

The mean participant processing time, from the initial date the research team sent out the recruitment pack to date of participant consent, was 18 days.

The mean number of physiotherapy appointments was 2.4 for the loaded self-managed group, over a mean duration of 3.1 months, compared with 3.2 appointments over 3.8 months for the comparator group.

Fidelity rate in the loaded self-managed group, measured by the three-point checklist was 95\% (Table 5). Measuring for contamination, this checklist recorded $0 \%$ in the usual physiotherapy group.

\section{Patient-reported outcome measures}

At three months follow-up, 44\% (4/9) of respondents in the loaded self-managed group and $39 \%(7 / 18)$ in the usual physiotherapy group were classified as recovered. At six months, 56\% (5/9) of respondents in the loaded self-managed group and 56\% (9/16) in the usual physiotherapy group were classified as recovered.

Both groups demonstrated improvements in average pain (VAS), kinesiophobia, pain catastrophizing, general self-efficacy and EQ-5D-5 L from baseline to six months (see Table 6).

\section{Embedded qualitative interviews}

\section{Recruitment and randomisation}

All participants' comments about the information sheets and letters sent to their home were positive, with no concerns raised.

"Yeah, they really helped me to decide [to take part]."

Also, participants were generally positive about the recruitment process to the feasibility $\mathrm{RCT}$, including 
Table 4 Baseline characteristics. Values are means (SD) unless stated otherwise

\begin{tabular}{lll}
\hline Characteristics & LSM Group $(n=30)$ & UP $(n=30)$ \\
\hline Age (years) & $31.4(7.1)$ & $27.4(6.6)$ \\
No of females (\%) & $15(50 \%)$ & $19(63 \%)$ \\
Duration of knee pain (months)* & $18(6.5-48)$ & $12(5-27)$ \\
Average pain VAS & $4.9(1.9)$ & $4.9(2.1)$ \\
TSK & $40.8(6.5)$ & $37.8(7.1)$ \\
PCS & $22.7(14.1)$ & $19.6(9.3)$ \\
GSES & $30.6(3.2)$ & $31.5(3.4)$ \\
EQ-5D-5 L* & $0.65(0.46-0.72)$ & $0.72(0.62-0.77)$ \\
Health VAS & $60.2(18.4)$ & $72.7(18.8)$ \\
Sport Participation* & $2.5(1-5)$ & $2(0.0-4)$ \\
\hline
\end{tabular}

*Median (interquartile range)

LSM loaded self-managed, UP usual physiotherapy, TSK Tampa Scale for Kinesiophobia, PCS Pain Catastrophizing Scale, GSES General Self Efficacy

Scale, EQ-5D-5 L EuroQol 5-dimensions, VAS visual analogue scale

receiving a telephone call, the appointment and randomisation process.

\section{Outcome measures}

Participants reflected upon appropriateness, ease of filling in and returning, and the time taken to complete the

Table 5 Treatments offered by physiotherapists

\begin{tabular}{|c|c|c|}
\hline \multirow[t]{2}{*}{$\begin{array}{l}\text { Possible physiotherapy } \\
\text { treatments }\end{array}$} & \multicolumn{2}{|c|}{$\begin{array}{l}\text { Treatments offered by treating } \\
\text { physiotherapist }\end{array}$} \\
\hline & $\begin{array}{l}\text { Loaded } \\
\text { Self-Managed }\end{array}$ & Usual Physiotherapy \\
\hline Specific pain education* & 23 & 0 \\
\hline Loaded exercise* & 26 & 0 \\
\hline Self-management strategies* & 25 & 0 \\
\hline Close chain exercise & 21 & 27 \\
\hline General advice & 21 & 20 \\
\hline Open chain exercise & 1 & 23 \\
\hline Orthotics & 1 & 0 \\
\hline Movement re-training & 0 & 11 \\
\hline Hip specific exercise & 0 & 9 \\
\hline Stretches & 0 & 3 \\
\hline VM exercises & 0 & 2 \\
\hline Referral on & 0 & 1 \\
\hline Electrotherapy & 0 & 0 \\
\hline Acupuncture & 0 & 0 \\
\hline Patella taping & 0 & 0 \\
\hline Massage & 0 & 0 \\
\hline Mobilisations & 0 & 0 \\
\hline Other & 0 & 0 \\
\hline
\end{tabular}

*, three-point checklist outlining important details and components of the intervention; VM, vastus medialis questionnaire booklets. All, patients were positive about them, with seven patients happy to give no feedback.

"Yeah. The questions, I mean, all seemed fairly normal. I wouldn't have any issues understanding what it was actually asking me or anything."

Three participants provided feedback about the clinical outcome measures, including recommendation on the addition of a text-box to write open text, if needed; less ambiguity about if the questions were being asked in relation to just their knee pain, or their whole body; wording of the physical activity questions that one patient felt implied they were not already physically active; and use of the word 'accident' in a patient group where the pain usually developed insidiously.

Five participants were contacted who had failed to return any outcome measures. All five initially agreed to be interviewed; unfortunately, four failed to attend. Of the ten patients who were interviewed, five returned all outcome measures, four returned one, and one patient failed to return any outcome measures. Of the patients who failed to return all of their outcome measures, four of them stated that they had, suggesting some problem with the pre-paid envelopes and return of the paperwork back to the physiotherapy department at the hospital.

However, the one participant who did acknowledge failing to return one of their outcome measure packs reported that it was due to forgetting, with some difficulty regarding living in different places as a university student.

"Think I might have forgotten it and then ended up leaving it at home before I came. Because I live in halls here."

\section{Overall study design}

Participants were also asked to reflect on the ways in which study design could be improved. In addition to the feedback already mentioned about the outcome measures, other improvement ideas were: the use of text message reminders (filling in and returning the outcome forms) and information concerning what would happen if they did not respond to treatment whilst in the study.

Finally, physiotherapists were asked to reflect upon, and discuss, their thoughts on the training package delivered at the start of the feasibility RCT. No major concerns or improvements were mentioned; however, the physiotherapists did reference the moderate frequency of other clinical trials running in the department, particularly ones incorporating pain science, suggesting a practised competence at interpreting new information and 
Table 6 Clinical outcomes. Mean (SD) unless otherwise stated

\begin{tabular}{|c|c|c|c|c|c|c|}
\hline Outcome & Group & Baseline (SD) & 3 months (SD) & 6 months (SD) & $\begin{array}{l}\text { Mean difference } \\
\text { (LSM-UP, } 6 \text { months) (95\% Cl) }\end{array}$ & $\begin{array}{l}\text { ES (d) } \\
\text { (LSM-UP, } 6 \text { months) }\end{array}$ \\
\hline \multirow[t]{2}{*}{ Average pain VAS } & LSM & $4.8(1.9)$ & $2.9(1.4)$ & $2.1(2.2)$ & $-1.2(-3.4,1.1)$ & 0.43 \\
\hline & UP & $4.8(2.1)$ & $3.0(2.8)$ & $2.4(2.6)$ & & \\
\hline \multirow[t]{2}{*}{ TSK } & LSM & $40.8(6.6)$ & $34.7(6.2)$ & $31.3(8.8)$ & $-4.3(-10.9,2.3)$ & 0.51 \\
\hline & UP & $38.3(7.1)$ & $34.1(8.6)$ & $31.6(6.3)$ & & \\
\hline \multirow[t]{2}{*}{ PCS } & LSM & $22.0(13.8)$ & $16.0(10.5)$ & $12.6(9.1)$ & $-4.0(-14.8,6.8)$ & 0.29 \\
\hline & UP & $20.0(9.4)$ & $16.6(13.5)$ & $14.1(10.4)$ & & \\
\hline \multirow[t]{2}{*}{ GSES } & LSM & $30.6(3.2)$ & $29.9(3.4)$ & $32.3(2.4)$ & $1.8(-1.0,4.6)$ & 0.53 \\
\hline & UP & $31.6(3.5)$ & $31.2(3.5)$ & $31.8(3.2)$ & & \\
\hline \multirow[t]{2}{*}{$E Q-5 D-5 L^{*}$} & LSM & $0.65(0.53-0.73)$ & $0.75(0.54-0.82)$ & $0.74(0.64-0.92)$ & & \\
\hline & UP & $0.71(0.62-0.79)$ & $0.80(0.69-1.00)$ & $0.84(0.73-0.87)$ & & \\
\hline \multirow[t]{2}{*}{ Health VAS } & LSM & $60.9(18.4)$ & $67.8(16.0)$ & $64.4(26.4)$ & $8.5(-8.1,25.2)$ & 0.51 \\
\hline & UP & $72.1(19.4)$ & $80.6(17.0)$ & $79.8(17.4)$ & & \\
\hline \multirow[t]{2}{*}{ Sport Participation* } & LSM & $3.0(1.0-5.0)$ & $2.0(1.5-4.0)$ & $3.0(2.0-6.0)$ & & \\
\hline & UP & $2.0(0.0-4.0)$ & $3.5(2.0-4.3)$ & $3.5(2.0-5.0)$ & & \\
\hline \multirow[t]{2}{*}{ GROC } & LSM & & $44.4 \%$ (4 / 9) & $55.6 \%(5 / 9)$ & & \\
\hline & UP & & $38.9 \%(7 / 18)$ & $56.3 \%(9 / 16)$ & & \\
\hline \multirow[t]{2}{*}{$\mathrm{GROC}^{* *}$} & LSM & & $100 \%(9 / 9)$ & $77.8 \%(7 / 9)$ & & \\
\hline & UP & & $77.8 \%(14 / 18)$ & $87.5 \%(14 / 16)$ & & \\
\hline
\end{tabular}

LSM loaded self-managed, UP usual physiotherapy, Cl confidence interval, TSK Tampa Scale for Kinesiophobia, PCS Pain Catastrophizing Scale, GSES General Self Efficacy Scale, EQ-5D-5 L EuroQol 5-dimensions, VAS visual analogue scale, GROC global rating of change scale, ES effect size ${ }^{*}$, Median (interquartile range); ${ }^{* *}$, sensitivity analysis

implementing into clinical practice, that departments not accustomed to clinical trials may find difficult.

"No, it wasn't a culture shock or anything that I felt I needed to kind of do very differently."

\section{Discussion}

The results of this study confirm that it is feasible and acceptable to deliver a loaded self-managed exercise programme to adults with PFP in an NHS physiotherapy outpatient setting. However, differences in lost to follow up and poor exercise diary completion mean we are uncertain on some feasibility aspects, with potential for systematic bias. Further feasibility work may be needed to address these issues, before supporting a larger clinical trial which will evaluate the clinical and cost-effectiveness of a loaded self-managed exercise programme for people with PFP compared with usual physiotherapy.

\section{Process}

The observed recruitment rate was 5.0 participants per month at the single site over 13 months. We had expected to be able to recruit 4.6 patients per month but deemed a recruitment rate of 3.7 feasible. Our initial recruitment strategy was an estimate based on the referral rate (after full screening by a physiotherapist) of 23 per month (based on our inclusion and exclusion criteria), which was observed in the department between January 2013 and October 2013. However, the observed number of referrals during the study was less than the rate observed in 2013, with 16.8 potentially eligible patients referred each month (before full screening). Therefore, any future definitive trial should consider a lower referral rate than we had initially anticipated.

Of the participants interviewed, both patients and physiotherapists, no barriers to recruitment were identified. However, as the embedded interviews were conducted on recruited participants, it may represent a biased sample. Furthermore, it may be that the participants interviewed felt inhibited to provide negative feedback because they were interviewed by the main researcher. From a management perspective, 50 (27.8\%) potential recruits were uncontactable via the telephone numbers supplied by the referring healthcare professional. Therefore, any future trial may need to include strategies in the protocol to cross-check potential eligible participants contact details on referrals with primary care databases.

The qualitative interviews identified no barriers or issues with the delivery of the training package to the physiotherapists. However, positive reference was made to the physiotherapy department's frequency in participating in clinical trials, suggesting the staff were more 
willing and able to adapt to the suggested evidence-based training package than, perhaps, a department less experienced in clinical trials.

\section{Resources}

One of the biggest challenges with exercise interventions is treatment adherence and monitoring of adherence. With unsupervised exercises, it is unclear to what degree participants have engaged with the prescribed exercise to obtain any therapeutic benefit. This feasibility study used exercise diaries as a measure of self-reported exercise adherence. The three diaries returned indicated exercise adherence of $42 \%$ of the time ( $40 \%$ in the loaded self-managed exercise group compared with $43 \%$ in the usual physiotherapy group). However, with such a low return rate, the reliability and validity of the data are limited. Recent systematic reviews have highlighted the lack of validated and reliable self-report measures for unsupervised, exercise-based rehabilitation adherence $[61,62]$. Therefore, any future definitive trial may need to consider it unfeasible to monitor adherence through self-reported paper-based measures.

Through qualitative interviews with both patients and physiotherapists, we were able to understand the acceptability of the study design and any potential challenges that may occur with implementation into a large RCT. Patients and physiotherapists perceived some value and benefit from the intervention, which can be seen in the effect sizes of the patient reported outcome measures (Table 6), though the degree and nature of this benefit were variable, with the aforementioned un-even lost to follow up.

\section{Management}

The fidelity assessment highlighted some interesting findings on current practice (Table 5). In contrast to current UK wide physiotherapy treatment, and international best practice guidelines $[9,50]$, very few physiotherapists provided movement retraining exercises, vastus medialis (VM) exercises, hip-specific exercise or stretches; and no physiotherapists in the usual physiotherapy group offered patella taping, joint mobilisations or orthotics. Further qualitative work trying to understand the nature of and impact of the content of the control group was conducted and published separately. Nonetheless, 95\% of patients received the intervention as described in the protocol, indicating intervention fidelity was not an issue in this study.

The overall level of missing data for the returned patient-reported outcome measures was negligible $(0.3 \%)$. However, the return rate was below the feasibility threshold, with a retention rate of $42 \%$. The telephone and postal reminder for non-returned questionnaires did not improve the response. The researcher also asked treating physiotherapists to prompt participants, should they still be receiving physiotherapy management; this accounted for four receipts of outcome questionnaires, each one being completed in the physiotherapy department during a follow-up appointment. It remains unclear why such a large lost to follow-up occurred. Patient representatives of the trial steering committee approved the completion time of the questionnaires, and this was confirmed by the qualitative interviews of the participants. Of the five patients interviewed who failed to return an outcome questionnaire, four stated they had, suggesting some problem with the pre-paid envelopes. However, internal and external testing of the pre-paid envelopes in the UK and hospital mail system operated correctly. One patient did acknowledge to having failed to return one of their outcome questionnaires saying they simply forgot. This compares to 97 and $96 \%$ (at 12 months) in the largest PFP RCT trials to date in the Netherlands and Australia respectively [51, 63]. These trials optimised collection of the main outcome measure by telephone follow-ups and e-mail, rather than relying solely on postal mail.

The retention rate was uneven, with 21 lost to follow-up (70\%) in the loaded self-managed group compared with 14 (47\%) in the usual physiotherapy group at six months. Of note, is the four participants who failed to return outcome forms at six months, who were still under the care of the physiotherapists and then went on to complete their six month outcome data during their follow-up appointments, were all in the usual physiotherapy group. One possible explanation for the uneven lost to follow-up rate could be the fewer appointments received and the shorter period under physiotherapy management that the loaded self-managed group received compared with the usual physiotherapy group. Ten per cent $(3 / 29)$ of participants in the loaded self-managed group were still under physiotherapy care at the six month follow-up, compared with $50 \%(14 / 28)$ in the usual physiotherapy group. Lost to follow-up is lower when participants outcome schedule occurs with appointments [64]. Indeed, the mean number of appointments were 2.4 and 3.2 in the loaded self-managed group and usual physiotherapy, respectively; this is considerably lower than the 7.7 appointments seen elsewhere in the UK [65]. A future definitive trial should make modifications to address participant engagement with the study, particularly after they have been discharged from physiotherapy. Strategies could include weekly telephone calls, frequent newsletters, and the use of e-mail or text messaging for measuring adherence or patient report outcome measures.

High participant attrition has been observed in different patient populations, in the same physiotherapy outpatients department, in a large scale RCT (56\% at six months) [24]. A high attrition rate does 
not necessarily mean that large-scale RCTs are unfeasible $[66,67]$, merely that the sample size calculation and recruitment rate should be adjusted to account for this. Further feasibility work may be warranted to test strategies to improve attrition, including, reducing the burden of the number of outcome measures; use of technology, e.g. text-messaging for reminders or using short outcome measures like pain score or GROC; asking the treating physiotherapists to remind the participants at three months; relaxing the criteria for success; using patient incentives to return forms, e.g. entry into a prize draw; using telephone consultations to complete the questionnaires; or telephoning participants at evenings and weekends [64]. Interestingly, feasibility studies looking at web-based patient questionnaires have found equally low return rates (33\% at 24 weeks follow-up), demonstrating the complexity of finding solutions to this problem.

\section{Strength and limitations}

The principle strengths of this research are the comprehensive use of a mixed-methods approach, which was based on the recommendations of the Medical Research Council for the evaluation of complex interventions [68]; the use of concealed random allocation; and its pragmatic evaluation of physiotherapy assessment and interventions.

Study limitations included, firstly, the research being conducted at a single centre, thus reducing its generalisability. Whilst baseline demographics, symptom duration and average pain scores were comparable to previous trials in PFP $[69,70]$; a definitive RCT would be required to be multicentred to improve generalisability [64]. Secondly, baseline patient-reported outcome measures were completed in front of an unblinded assessor. Future trials should introduce blinded assessors to reduce the risk of bias [64].

As briefly mentioned above, and despite efforts to the contrary, the embedded qualitative component was likely to have produced a biased sample of participants who had largely positive feelings towards the design of the study. Furthermore, they knew they were being interviewed by the main researcher and therefore may have felt obliged to answer questions favourably. Strategies to reduce this risk such as conducting interviews with an independent researcher or collecting anonymised questionnaires may be considered.

Lastly, as the exercise intervention was considered low risk, being commonly used in musculoskeletal pain populations, no adverse event data were collected.

\section{Conclusion}

A loaded self-managed exercise programme designed around: pain education; a loaded exercise programme; and self-management strategies, is feasible and acceptable to deliver in an NHS physiotherapy outpatient setting. However, the present study demonstrates that, even with the appropriate physiotherapist training package, further feasibility work may be needed to address differences in lost to follow up and poor diary completion. These methodological issues need addressing, before supporting a larger clinical trial which will evaluate the clinical and cost-effectiveness of a loaded self-managed exercise programme for people with PFP compared with usual physiotherapy.

\section{Abbreviations \\ Cl: Confidence interval; CONSORT: Consolidate Standard of Reporting Trials; DNA: Did not attend; EQ-5D-5 L: Euro-QOL; ES: Cohen's effect size; GROC: Global rating of change; GSES: General self-efficacy scale; HEE: Health Education England; LSM: Loaded self-managed; MSK: Musculoskeletal; NHS: National Health Service; NIHR: National Institute for Health Research; PCS: Pain catastrophising scale; PFP: Patellofemoral pain; RCT: Randomised controlled trial; SD: Standard deviation; TiDieR: Template for Intervention Description and Replication guidelines; TSK: Tampa Scale for Kinesiophobia; UP: Usual physiotherapy; VAS: Visual analogue scale; VM: Vastus medialis}

\section{Acknowledgements}

We acknowledge the staff of the physiotherapy outpatient department at London Road Community Hospital, Derby, who treated the patients in this study.

We also acknowledge Katherine Walstow and Matthew Stuart who kindly helped with data collection, and Jo-Fen Liu, School of Medicine, University of Nottingham; who helped with the statistical analysis.

\section{Funding}

This report is independent research arising from a Clinical Doctoral Research Fellowship, Benjamin Smith, ICA-CDRF-2015-01-002 supported by the National Institute for Health Research (NIHR) and Health Education England (HEE).

Dr. Toby Smith is supported by the National Institute for Health Research (NIHR) Biomedical Research Centre, Oxford.

The views expressed in this publication are those of the author(s) and not necessarily those of the NHS, the NIHR or the Department of Health and Social Care.

The funding body have played no role in the design of the study and collection, analysis, and interpretation of data and in writing the manuscript.

\section{Availability of data and materials}

The datasets used and/or analysed during the current study is available from the corresponding author on reasonable request.

\section{Authors' contributions}

BES was the chief investigator, he collected the data, analysed the data, and drafted the manuscript. PH, MB, FM, MSR, JS, TOS and PL were study investigators who participated in the development of the study protocol. All authors contributed to the editing and revising of the manuscript. All authors read and approved the final manuscript.

\section{Ethics approval and consent to participate}

This study was approved by the West Midlands - Black Country Research Ethics Committee (16/WM/0414 IRAS reference 211,417$)$ and Sponsored by University Hospitals of Derby and Burton NHS Foundation Trust. IRAS reference 211417.

Written consent to participate in the study was obtained from the participants.

\section{Consent for publication}

Not applicable.

\section{Competing interests}

The authors declare that they have no competing interests. 


\section{Publisher's Note}

Springer Nature remains neutral with regard to jurisdictional claims in published maps and institutional affiliations.

\begin{abstract}
Author details
${ }^{1}$ Physiotherapy Department (Level 3) London Road Community Hospital, University Hospitals of Derby and Burton NHS Foundation Trust, Derby DE1 2QY, UK. ²Division of Rehabilitation and Ageing, School of Medicine, University of Nottingham, Nottingham, UK. ${ }^{3}$ Division of Physiotherapy and Rehabilitation Sciences, School of Health Sciences, University of Nottingham, Nottingham University Hospitals (City Campus), Nottingham, UK. ${ }^{4}$ Research Unit for General Practice in Aalborg, Department of Clinical Medicine, Aalborg University, Aalborg, Denmark. ${ }^{5}$ Department of Occupational Therapy and Physiotherapy, Department of Clinical Medicine, Aalborg University Hospital, Aalborg, Denmark. ${ }^{6}$ Department of Health Professions, Manchester Metropolitan University, Manchester, UK. ${ }^{7}$ Nuffield Department of Orthopaedics, Rheumatology and Musculoskeletal Sciences, University of Oxford, Oxford, UK.
\end{abstract}

\section{Received: 12 November 2018 Accepted: 17 March 2019 Published online: 27 March 2019}

\section{References}

1. Dey P, Callaghan M, Cook N, Sephton R, Sutton C, Hough E, et al. A questionnaire to identify patellofemoral pain in the community: an exploration of measurement properties. BMC Musculoskelet Disord. 2016;17: 237. https://doi.org/10.1186/s12891-016-1097-5.

2. Urwin M, Symmons D, Allison T, Brammah T, Busby H, Roxby M, et al. Estimating the burden of musculoskeletal disorders in the community: the comparative prevalence of symptoms at diverent anatomical sites, and the relation to social deprivation. Ann Rheum Dis. 1998;57:649-55.

3. Jordan KP, Kadam UT, Hayward R, Porcheret M, Young C, Croft P, et al. Annual consultation prevalence of regional musculoskeletal problems in primary care: an observational study. BMC Musculoskelet Disord. 2010;11: 144. https://doi.org/10.1186/1471-2474-11-144.

4. Smith BE, Selfe J, Thacker D, Hendrick P, Bateman M, Moffatt F, et al. Incidence and prevalence of patellofemoral pain: a systematic review and meta-analysis. PLoS One. 2018;13(1). https://doi.org/10.1371/journal. pone.0190892.

5. Collins NJ, Bierma-Zeinstra SMA, Crossley KM, van Linschoten RL, Vicenzino B, van Middelkoop M, et al. Prognostic factors for patellofemoral pain: a multicentre observational analysis. Br J Sports Med. 2013;47:227-33. https:// doi.org/10.1136/bjsports-2012-091696.

6. Stathopulu E, Baildam E. Anterior knee pain: a long-term follow-up. Rheumatology (Oxford). 2003;42:380-2.

7. Doménech J, Sanchis-Alfonso V, Espejo BBB, Domenech J, Sanchis-Alfonso $V$, Espejo BBB. Changes in catastrophizing and kinesiophobia are predictive of changes in disability and pain after treatment in patients with anterior knee pain. Knee Surg Sports Traumatol Arthrosc. 2014;22:2295-300. https:// doi.org/10.1007/s00167-014-2968-7.

8. Clijsen R, Fuchs J, Taeymans J. Effectiveness of exercise therapy in treatment of patients with patellofemoral pain syndrome: systematic review and metaanalysis. Phys Ther. 2014;94:1697-708. https://doi.org/10.2522/ptj.20130310.

9. Barton CJ, Lack S, Hemmings S, Tufail S, Morrissey D. The "best practice guide to conservative Management of Patellofemoral Pain": incorporating level 1 evidence with expert clinical reasoning. Br J Sports Med. 2015;49: 923-34. https://doi.org/10.1136/bjsports-2014-093637.

10. van der Heijden RA, Lankhorst NE, van Linschoten R, Bierma-Zeinstra SMA, van Middelkoop M. Exercise for treating patellofemoral pain syndrome. Cochrane Database Syst Rev. 2015;1:CD010387. https://doi.org/10.1002/ 14651858.CD010387.pub2.

11. Doménech J, Sanchis-Alfonso V, López L, Espejo BB, Domenech J, SanchisAlfonso $\mathrm{V}$, et al. Influence of kinesiophobia and catastrophizing on pain and disability in anterior knee pain patients. Knee Surgery, Sport Traumato Arthrosc. 2013;21:1562-8. https://doi.org/10.1007/s00167-012-2238-5.

12. Piva SR, Fitzgerald GK, Wisniewski S, Delitto A. Predictors of pain and function outcome after rehabilitation in patients with patellofemoral pain syndrome. J Rehabil Med. 2009;41:604-12. https://doi.org/10.2340/ 16501977-0372.

13. Piva SR, Fitzgerald GK, Irrgang JJ, Fritz JM, Wisniewski S, McGinty GT, et al. Associates of Physical Function and Pain in patients with patellofemoral pain syndrome. Arch Phys Med Rehabil. 2009;90:285-95. https://doi.org/10. 1016/j.apmr.2008.08.214.

14. Smith BE, Moffatt F, Hendrick P, Bateman M, Rathleff MS, Selfe J, et al. The experience of living with patellofemoral pain-loss, confusion and fearavoidance: a UK qualitative study. BMJ Open. 2018;8:e018624. https://doi. org/10.1136/bmjopen-2017-018624.

15. Ossipov MH, Dussor GO, Porreca F. Central modulation of pain. J Clin Invest. 2010;120:3779.

16. Quartana PJ, Campbell CM, Edwards RR. Pain catastrophizing: a critical review. Expert Rev Neurother. 2009;9:745-58. https://doi.org/10.1586/ern.09.34

17. Moseley GL, Arntz A. The context of a noxious stimulus affects the pain it evokes. Pain. 2007;133:64-71.

18. Harvie DS, Broecker M, Smith RT, Meulders A, Madden VJ, Moseley GL. Bogus visual feedback alters onset of movement-evoked pain in people with neck pain. Psychol Sci. 2015:0956797614563339.

19. Lobanov OV, Zeidan F, McHaffie JG, Kraft RA, Coghill RC. From cue to meaning: brain mechanisms supporting the construction of expectations of pain. Pain. 2014;155:129-36.

20. Chou R, Shekelle $P$. Will this patient develop persistent disabling low back pain? JAMA. 2010;303:1295-302. https://doi.org/10.1001/jama. 2010.344

21. Moseley GL. Reconceptualising pain according to modern pain science. Phys Ther Rev. 2007:12:169-78. https://doi.org/10.1179/108331907X223010.

22. Miles CL, Pincus T, Carnes D, Homer KE, Taylor SJC, Bremner SA, et al. Can we identify how programmes aimed at promoting selfmanagement in musculoskeletal pain work and who benefits? A systematic review of sub-group analysis within RCTs. Eur J Pain. 2011; 15. https://doi.org/10.1016/j.ejpain.2011.01.016.

23. Ohberg $L$, Alfredson $H$. Effects on neovascularisation behind the good results with eccentric training in chronic mid-portion Achilles tendinosis? Knee Surg Sports Traumatol Arthrosc. 2004;12:465-70. https://doi.org/10. 1007/s00167-004-0494-8.

24. Littlewood C, Bateman M, Brown K, Bury J, Mawson S, May S, et al. A selfmanaged single exercise programme versus usual physiotherapy treatment for rotator cuff tendinopathy: a randomised controlled trial (the SELF study). Clin Rehabil. 2016:30:686-96. https://doi.org/10.1177/0269215515593784.

25. Bernhardsson S, Klintberg $1 \mathrm{H}$, Wendt GK. Evaluation of an exercise concept focusing on eccentric strength training of the rotator cuff for patients with subacromial impingement syndrome. Clin Rehabil. 2011;25:69-78. https:// doi.org/10.1177/0269215510376005.

26. Holmgren $T$, Bjornsson Hallgren $H$, Oberg B, Adolfsson L, Johansson $K$, Björnsson Hallgren $\mathrm{H}$, et al. Effect of specific exercise strategy on need for surgery in patients with subacromial impingement syndrome: randomised controlled study. BMJ. 2012;344:e787. https://doi.org/10. 1136/bmj.e787

27. Long A, Donelson R, Fung T. Does it matter which exercise? A randomized control trial of exercise for low back pain. Spine (Phila Pa 1976). 2004;29:2593-602

28. Cook C, Hegedus EJ, Ramey K. Physical therapy exercise intervention based on classification using the patient response method: a systematic review of the literature. J Man Manip Ther. 2005;13:152-62. https://doi.org/10.1179/ 106698105790824950

29. Rathleff MS, Molgaard CM, Fredberg U, Kaalund S, Andersen KB, Jensen TT, et al. High-load strength training improves outcome in patients with plantar fasciitis: a randomized controlled trial with 12-month follow-up. Scand J Med Sci Sports. 2015;25:e292-300. https://doi.org/10.1111/sms.12313.

30. Smith BE, Hendrick P, Bateman M, Holden S, Littlewood C, Smith TO, et al. Musculoskeletal pain and exercise - challenging existing paradigms and introducing new. Br J Sports Med. 2018. https://doi.org/10.1136/ bjsports-2017-098983.

31. Smith BE, Hendrick P, Smith TO, Bateman M, Moffatt F, Rathleff MS, et al. Should exercises be painful in the management of chronic musculoskeletal pain? A systematic review and meta-analysis. Br J Sports Med. 2017;51:167987. https://doi.org/10.1136/bjsports-2016-097383.

32. Osteras $\mathrm{B}$, Osteras $\mathrm{HH}$, Torstensen TA, Vasseljen $\mathrm{O}$, Østerås $\mathrm{B}$, Østerås $\mathrm{H}$, et al. Dose-response effects of medical exercise therapy in patients with patellofemoral pain syndrome: a randomised controlled clinical trial. Physiotherapy. 2013;99:126-31. https://doi.org/10.1016/j.physio.2012.05.009.

33. Rathleff MS, Roos EM, Olesen JL, Rasmussen S. Exercise during school hours when added to patient education improves outcome for 2 years in adolescent patellofemoral pain: a cluster randomised trial. Br J Sports Med. 2015;49:406-12. https://doi.org/10.1136/bjsports-2014-093929. 
34. Schulz KF, Altman DG, Moher D. CONSORT 2010 statement: updated guidelines for reporting parallel group randomised trials. BMC Med. 2010;8:18.

35. Hoffmann TC, Glasziou PP, Boutron I, Milne R, Perera R, Moher D, et al. Better reporting of interventions: template for intervention description and replication (TIDieR) checklist and guide. Bmj. 2014;348:g1687.

36. Smith BE, Hendrick P, Bateman M, Moffatt F, Rathleff MS, Selfe J, et al. Study protocol: a mixed methods feasibility study for a loaded self-managed exercise programme for patellofemoral pain. Pilot Feasibility Stud. 2017;4. https://doi.org/10.1186/s40814-017-0167-2.

37. Crossley KM, Stefanik JJ, Selfe J, Collins NJ, Davis IS, Powers CM, et al. 2016 Patellofemoral pain consensus statement from the 4th International Patellofemoral Pain Research Retreat, Manchester. Part 1: Terminology, definitions, clinical examination, natural history, patellofemoral osteoarthritis and patient-reported outcome m. Br J Sports Med 2016:50:839-843.

38. Julious SA. Sample size of 12 per group rule of thumb for a pilot study. Pharm Stat J Appl Stat Pharm Ind. 2005:4:287-91.

39. Sim J, Lewis M. The size of a pilot study for a clinical trial should be calculated in relation to considerations of precision and efficiency. J Clin Epidemiol. 2012;65:301-8.

40. Arain M, Campbell MJ, Cooper CL, Lancaster GA. What is a pilot or feasibility study? A review of current practice and editorial policy. BMC Med Res Methodol. 2010;10:67. https://doi.org/10.1186/1471-2288-10-67.

41. Smith BE, Littlewood C, May S. An update of stabilisation exercises for low back pain: a systematic review with meta-analysis. BMC Musculoskelet Disord. 2014;15:416. https://doi.org/10.1186/1471-2474-15-416.

42. Loudon JK, Wiesner D, Goist-Foley HL, Asjes C, Loudon KL. Intrarater reliability of functional performance tests for subjects with patellofemoral pain syndrome. J Athl Train. 2002;37:256-61.

43. Littlewood C, Ashton J, Mawson S, May S, Walters S. A mixed methods study to evaluate the clinical and cost-effectiveness of a self-managed exercise programme versus usual physiotherapy for chronic rotator cuff disorders: protocol for the SELF study. BMC Musculoskelet Disord. 2012;13: 62. https://doi.org/10.1186/1471-2474-13-62.

44. Littlewood C. Contractile dysfunction of the shoulder (rotator cuff tendinopathy): an overview. J Man Manip Ther. 2012;20:209-13. https://doi. org/10.1179/2042618612Y.0000000005.

45. McKenzie R, May S. The human extremities: Mechanical Diagnosis \& Therapy. Waikanee. New Zealand: Spinal Publications; 2000.

46. McLean SM, Burton M, Bradley L, Littlewood C. Interventions for enhancing adherence with physiotherapy: a systematic review. Man Ther. 2010;15:51421. https://doi.org/10.1016/j.math.2010.05.012.

47. Littlewood C, Malliaras P, Mawson S, May S, Walters S. Development of a selfmanaged loaded exercise programme for rotator cuff tendinopathy. Physiother (United Kingdom). 2013;99:358-62. https://doi.org/10.1016/.jphysio.2012.12.002.

48. Hoffman AJ. Enhancing self-efficacy for optimized patient outcomes through the theory of symptom self-management. Cancer Nurs. 2013;36: E16-26. https://doi.org/10.1097/NCC.0b013e31824a730a.

49. Nijs J, Lluch Girbés E, Lundberg M, Malfliet A, Sterling M. Exercise therapy for chronic musculoskeletal pain: innovation by altering pain memories. Man Ther. 2015;20:216-20. https://doi.org/10.1016/j.math.2014.07.004.

50. Smith BE, Hendrick P, Bateman M, Moffatt F, Rathleff MS, Selfe J, et al. Current management strategies for patellofemoral pain: an online survey of 99 practising UK physiotherapists. BMC Musculoskelet Disord. 2017;18. https://doi.org/10.1186/s12891-017-1539-8

51. van Linschoten R, van Middelkoop M, Berger MY, Heintjes EM, Verhaar JAN, Willemsen SP, et al. Supervised exercise therapy versus usual care for patellofemoral pain syndrome: an open label randomised controlled trial. BMJ. 2009;339:b4074.

52. Rathleff MS, Roos EM, Olesen JL, Rasmussen S. Early intervention for adolescents with patellofemoral pain syndrome--a pragmatic cluster randomised controlled trial. BMC Musculoskelet Disord. 2012;13:9. https:// doi.org/10.1186/1471-2474-13-9.

53. Price DD, McGrath PA, Rafii A, Buckingham B. The validation of visual analogue scales as ratio scale measures for chronic and experimental pain. Pain. 1983;17:45-56.

54. Kori S, Miller R, Odd D. Kinesiophobia: a new view of chronic pain behaviour. Pain Manag. 1990;3:35-43.

55. Sullivan MJL, Bishop SR, Pivik J. The pain catastrophizing scale: development and validation. Psychol Assess. 1995;7:524-32. https://doi. org/10.1037/1040-3590.7.4.524.
56. Schwarzer R, Jerusalem M. Generalized self-efficacy scale. Meas Heal Psychol A User's Portfolio Causal Control Beliefs. 1995;1:35-7.

57. Devlin NJ, Krabbe PFM. The development of new research methods for the valuation of EQ-5D-5L. Eur J Health Econ. 2013;14:1.

58. Lancaster GA, Dodd S, Williamson PR. Design and analysis of pilot studies: recommendations for good practice. J Eval Clin Pract. 2004;10:307-12.

59. Moher D, Hopewell S, Schulz KF, Montori V, Gøtzsche PC, Devereaux PJ, et al. CONSORT 2010 explanation and elaboration: updated guidelines for reporting parallel group randomised trials. Int J Surg. 2012;10:28-55.

60. Braun V, Clarke V. Using thematic analysis in psychology. Qual Res Psychol. 2006:3:77-101.

61. Uzawa H, Davis S. Outcome measures for adherence to home exercises among patients with chronic low back pain: a systematic review. J Phys Ther Sci. 2018;30:649-53.

62. Bollen JC, Dean SG, Siegert RJ, Howe TE, Goodwin VA. A systematic review of measures of self-reported adherence to unsupervised home-based rehabilitation exercise programmes, and their psychometric properties. BMJ Open. 2014;4:e005044

63. Collins N, Crossley K, Beller E, Darnell R, McPoil T, Vicenzino B. Foot orthoses and physiotherapy in the treatment of patellofemoral pain syndrome: randomised clinical trial (reprinted from BMJ, vol 337, pg a1735, 2008). Br J Sports Med. 2009:43:169-U30.

64. Karanicolas PJ, Farrokhyar F, Bhandari M. Blinding: who, what, when, why, how? Can J Surg. 2010;53:345.

65. Brown J. Physiotherapists' knowledge of patello-femoral pain syndrome. Br J Ther Rehabil. 2000;7:346-54. https://doi.org/10.12968/bjtr.2000.7.8.13861.

66. Furlan AD, Pennick V, Bombardier C, van Tulder M, Editorial Board, Cochrane Back Review Group. 2009 updated method guidelines for systematic reviews in the Cochrane Back review group. Spine (Phila Pa 1976) 2009;34: 1929-1941. doi:https://doi.org/10.1097/BRS.0b013e3181b1c99f.

67. van Tulder MW, Suttorp M, Morton S, Bouter LM, Shekelle P. Empirical evidence of an association between internal validity and effect size in randomized controlled trials of low-back pain. Spine (Phila Pa 1976). 2009; 34:1685-92

68. Craig P, Dieppe P, Macintyre S, Mitchie S, Nazareth I, Petticrew M Developing and evaluating complex interventions: the new Medical Research Council guidance. BMJ. 2008;337:979-83. https://doi.org/10. 1136/bmj.a1655.

69. Selfe J, Janssen J, Callaghan M, Witvrouw E, Sutton C, Richards J, et al. Are there three main subgroups within the patellofemoral pain population? A detailed characterisation study of 127 patients to help develop targeted intervention (TIPPS). Br J Sport Med. 2016;50:873-80.

70. Drew BT, Conaghan PG, Smith TO, Selfe J, Redmond AC. The effect of targeted treatment on people with patellofemoral pain: a pragmatic, randomised controlled feasibility study. BMC Musculoskelet Disord. 2017;18:338.

Ready to submit your research? Choose BMC and benefit from:

- fast, convenient online submission

- thorough peer review by experienced researchers in your field

- rapid publication on acceptance

- support for research data, including large and complex data types

- gold Open Access which fosters wider collaboration and increased citations

- maximum visibility for your research: over $100 \mathrm{M}$ website views per year

At $\mathrm{BMC}$, research is always in progress.

Learn more biomedcentral.com/submission 